\title{
Efficacy of modified bevacizumab-XELOX therapy in Japanese patients with stage IV recurrent or non-resectable colorectal cancer
}

\author{
Daiki Yokoyama $^{1}$, Masaya Mukai ${ }^{1}$, Shuji Uda ${ }^{1}$, Kyouko Kishima ${ }^{1}$, Takuya Koike ${ }^{1}$, Sayuri Hasegawa ${ }^{1}$, \\ Hideki Izumi ${ }^{1}$, Souichirou Yamamoto ${ }^{1}$, Takayuki Tajima ${ }^{2}$, Eiji Nomura ${ }^{1}$, Hiroyasu Makuuchi ${ }^{1}$ \\ ${ }^{1}$ Department of Surgery, Tokai University Hachioji Hospital, Hachioji, Tokyo, Japan; ${ }^{2}$ Department of Surgery, Tokai University Tokyo Hospital, \\ Shibuya, Tokyo, Japan \\ Contributions: (I) Conception and design: D Yokoyama, M Mukai; (II) Administrative support: T Tajima, E Nomura, H Makuuchi; (III) Provision \\ of study materials or patients: S Uda, T Koike, S Hasegawa, H Izumi; (IV) Collection and assembly of data: D Yokoyama, S Yamamoto; (V) Data \\ analysis and interpretation: None; (VI) Manuscript writing: All authors; (VII) Final approval of manuscript: All authors. \\ Correspondence to: Daiki Yokoyama, MD. Lecturer in Surgery, Department of Surgery, Tokai University Hachioji Hospital, 1838 Ishikawa-machi, \\ Hachioji, 192-0032, Tokyo, Japan. Email: daiki66.d@gmail.com.
}

Background: Neoadjuvant chemotherapy (NAC) has been conducted for patients with non-resectable colorectal cancer; however, few reports of a systematic approach to NAC exist. At our hospital, bevacizumab with capecitabine and oxaliplatin (B-mab XELOX) has been used as chemotherapy for Stage IV colorectal cancer since 2014. We aimed to evaluate the efficacy and safety of NAC with a molecular-targeting agent for Stage IV colorectal cancer.

Methods: A retrospective, single-institute analysis was performed including 27 patients with advanced recurrent cancer following primary tumor resection and 43 patients with non-resectable tumors and remote metastasis. At the time of resection, 17 were receiving chemotherapy. All 70 patients received at least 3 cycles of B-mab XELOX (total: 920 cycles). We determined the 1-year progression-free survival (1Y-PFS), 1-year overall survival (1Y-OS), 3Y-PFS, 3Y-OS, and number of treatment cycles. The objective response rate, clinical benefit rate, and adverse events were assessed. The number of chemotherapy cycles, survival time, and R0 surgery rate were determined for patients who underwent RO conversion surgery.

Results: The 1Y-PFS was 28.5\% [median survival time (MST): 7.4 months], 1Y-OS was 76.6\% (MST not reached), 3Y-PFS was 5.5\% (MST: 7.4 months), and 3Y-OS was 26.4\% (MST: 25.2 months). The mean and median number of cycles of B-mab XELOX was 13.1 and 10.5, respectively. The objective response rate was $28.6 \%$, and the clinical benefit rate was 58.6\%. Grade 1 or Grade 2 adverse events occurred in 60 patients $(85.7 \%)$; however, they all resolved without intervention. A single Grade 4 event (perforation of the primary tumor) occurred in 1 patient $(1.4 \%)$. RO conversion surgery was performed in 7 patients $(10.0 \%$; primary + liver in 2 patients, primary + lung in 1 patient, liver in 3 patients, and primary in 1 patient). These patients received 3 to 10 cycles preoperatively (mean: 7.3; median: 6.5). R0 surgery was achieved in 5 of the 7 patients (71.4\%). Postoperative survival ranged from 1 to 26 months (MST: 8 months).

Conclusions: This modified regimen was safe and effective in Japanese patients, and a high quality of life/quality-adjusted life-year was achieved. To further evaluate PFS and OS, more patients are being investigated.

Keywords: Colorectal cancer; stage IV; chemotherapy; bevacizumab; XELOX; recurrent/non-resectable cancer

Submitted Aug 25, 2020. Accepted for publication Feb 15, 2021.

doi: 10.21037/jgo-20-350

View this article at: http://dx.doi.org/10.21037/jgo-20-350 


\section{Introduction}

Many molecular-targeting drugs have recently been developed and are used to treat advanced and recurrent colorectal cancer. These include vascular endothelial growth factor (VEGF) inhibitors (bevacizumab, ramucirumab, and VEGF-targeting fusion protein) and epidermal growth factor receptor (EGFR) inhibitors (panitumumab and cetuximab) for wild-type RAS/BRAF tumors (1-5) VEGF inhibitor therapy is particularly recommended for colorectal cancer originating from the midgut. However, BRAF V600E cancer incidence is high, and these tumors are larger than those arising from the left colon; therefore, the prognosis is generally poor (6-8). EGFR expression is high in left-sided colorectal cancer originating from the hindgut, including the rectum, and VEGF inhibitors are reported to improve survival significantly $(9,10)$. Furthermore, a significant proportion of right-sided tumors have the CpG island methylator phenotype and /or microsatellite instability, whereas chromosomal instability is more common among left-sided colorectal cancer. Thus, the biological features of colorectal cancer show regional differences $(7,8)$. Chemotherapy for Stage IV colorectal cancer has made considerable advances since m-FOLFOX/ FOLFIRI, although ramucirumab and ziv-aflibercept combined with FOLFIRI is used as second-line therapy, and most Phase III clinical studies used bevacizumab, for which genetic assay is not required $(1,5)$. Recently, neoadjuvant chemotherapy (NAC) has been conducted for patients with non-resectable colorectal cancer, and conversion surgery is also performed $(11,12)$. However, chemotherapy regimens that enable conversion surgery have numerous restricting conditions regarding metastatic lesions, and very few reports exist that present a comprehensive understanding as well as a unified approach.

Therefore, this retrospective study was performed to evaluate the efficacy and safety of NAC with a moleculartargeting agent for Stage IV colorectal cancer.

We present the following article in accordance with the STROBE reporting checklist (available at http://dx.doi. org/10.21037/jgo-20-350).

\section{Methods}

In patients with Stage IV colorectal cancer and remote metastasis in the liver and/or lungs, we preferentially performed surgical resection if $\mathrm{R} 0$ resection of the primary or metastatic tumors was macroscopically feasible, followed by adjuvant chemotherapy. When $\mathrm{R} 0$ resection was not feasible, chemotherapy was the first-line treatment, and R0 conversion surgery (R0-CS) was always attempted. Patients were followed with ultrasound (US)/CT imaging evaluation every 3-4 months for 5 years after surgery.

The diagnosis of primary colorectal cancer was made by histopathological examination of biopsy specimens obtained at colonoscopy. Metastasis to the liver and lungs was evaluated by diagnostic imaging, including US or CT and / or MRI or PET.

As Japanese patients have a smaller physique than western patients, if the original B-mab XELOX regimen is administered (oxaliplatin $130 \mathrm{mg} / \mathrm{m}^{2}$ every 3 weeks) Grade 3 adverse reactions, such as hand-foot syndrome and/or peripheral neuropathy, occur at a high frequency. A previous study on the original B-mab XELOX regimen showed that the incidence of hand-foot syndrome was $17 \%$ for Grade 3 and $1.7 \%$ for Grade 4 (13). Consequently, it is often difficult to continue treatment beyond 3 cycles (14-16). Accordingly, the dosages for FOLFOX4/6 (oxaliplatin $85 \mathrm{mg} / \mathrm{m}^{2}(17,18)$ and B-mab $5 \mathrm{mg} / \mathrm{kg}$ ) commonly used in Japan were administered as a 2 weeks on/2 weeks off cycle, or a 4-week cycle, on an outpatient basis. In addition, capecitabine $\left(2,000 \mathrm{mg} / \mathrm{m}^{2}\right)$ was administered orally in a 2 weeks on/2 weeks off cycle to complete the modified B-mab XELOX regimen (Figure 1) (17). This regimen was approved as NAC for Stage IV colorectal cancer by the Chemotherapy Review Committee of Tokai University Hachioji Hospital and the Institutional Review Board (IRB) of Tokai University Medical School. The protocol for the present retrospective study was also approved (IRB No. 18R-310). Of the 82 patients who have received the modified B-mab XELOX regimen as first-line treatment, a final 70 patients were evaluated. Patients were not excluded if they did not receive the intended 3 cycles; however, all 70 included did receive at least 3 cycles (total: 920 cycles). Patients with remote metastatic lymph nodes were also excluded from this study.

\section{Outcomes}

(I) One-year progression-free survival (1Y-PFS), 1-year overall survival (1Y-OS), $3 \mathrm{Y}-\mathrm{PFS}$, and $3 \mathrm{Y}-\mathrm{OS}$ were calculated from the first day of B-mab XELOX. The number of cycles was also determined.

(II) The RECIST Guideline (version 1.1) was used for assessment of response, and the objective response rate $(\mathrm{CR}+\mathrm{PR})$, clinical benefit rate $(\mathrm{CR}+\mathrm{PR}+\mathrm{SD}$ 
$\geq 6$ months), dose reduction rate, and dosing interval prolongation rate were calculated every 3-4 months (19).

(III) Adverse events during the initial 3 cycles of chemotherapy were evaluated by using CTCAE version 5 (20).

(IV) The number of chemotherapy cycles before R0 conversion surgery, survival time, and the R0 conversion rate were determined.

\section{Statistical analysis}

Statistical analysis was conducted using SPSS version 25 for Windows (IBM, Armonk, NY, USA). The event day was defined as the day when stable disease changed to progressive disease (PD) on ultrasound and CT scan as calculated from the first day of the first cycle. The 1Y-PFS, 1 Y-OS, 3Y-PFS, 3Y-OS, and median survival time (MST) were calculated.

Stage IV recurrent or non-resectable colorectal cancer

Modified-Bevacizumab + XELOX (Xeloda/Oxaliplatin) regimen

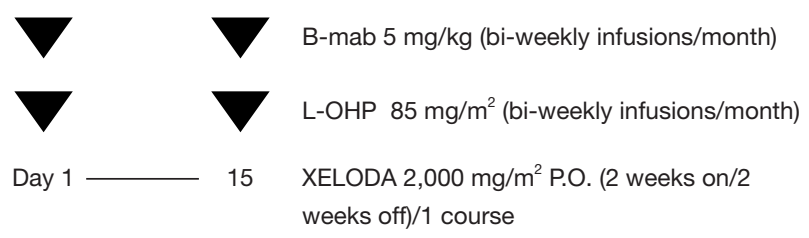

Figure 1 Modified B-mab XELOX regimen. Similar to FOLFOX 6 , oxaliplatin $\left(85 \mathrm{mg} / \mathrm{m}^{2}\right)$ is administered at the outpatient department on days 1 and 15 (one cycle $=4$ weeks). Capecitabine is administered for 2 weeks, followed by 2 weeks off therapy.

\section{Ethical statement}

The study was conducted in accordance with the Declaration of Helsinki (as revised in 2013). The study was approved by institutional/regional/national ethics/ committee/ethics board of Tokai University Medical School (No. 18R-310) and individual consent for this retrospective analysis was waived.

\section{Results}

Since 2009, we have employed various regimens containing bevacizumab (B-mab) as chemotherapy for Stage IV colorectal cancer, conducting a total of 2,466 cycles in 190 patients. Since April 2014, B-mab + capecitabine and oxaliplatin (XELOX) have been used systemically, and 82 patients have received a total of 941 cycles.

In this study, a total of 70 patients were evaluated. They comprised 27 patients (38.6\%) with Stage IV recurrent colorectal cancer following resection of the primary tumor and 43 patients $(61.4 \%)$ with non-resectable Stage IV colorectal cancer who had metastasis to the liver and/or lungs at diagnosis (Figure 2). Of the 27 patients with recurrent cancer, 19 patients had metachronous metastases (Stage I: 2 patients, Stage II: 5 patients, and Stage III: 12 patients). Seventeen patients were receiving chemotherapy at the time of the first resection (Stage II: 1 patient and Stage III: 16 patients). The original histopathological stage of the 27 patients with recurrence after curative resection was Stage I in 2 patients (7.4\%), Stage II in 5 patients (18.5\%), and Stage III in 20 patients (74.1\%) (Figure 2). In 13 of the 43 patients with unresectable disease $(30.2 \%)$, only the primary tumor was resected. Four patients $(9.3 \%)$

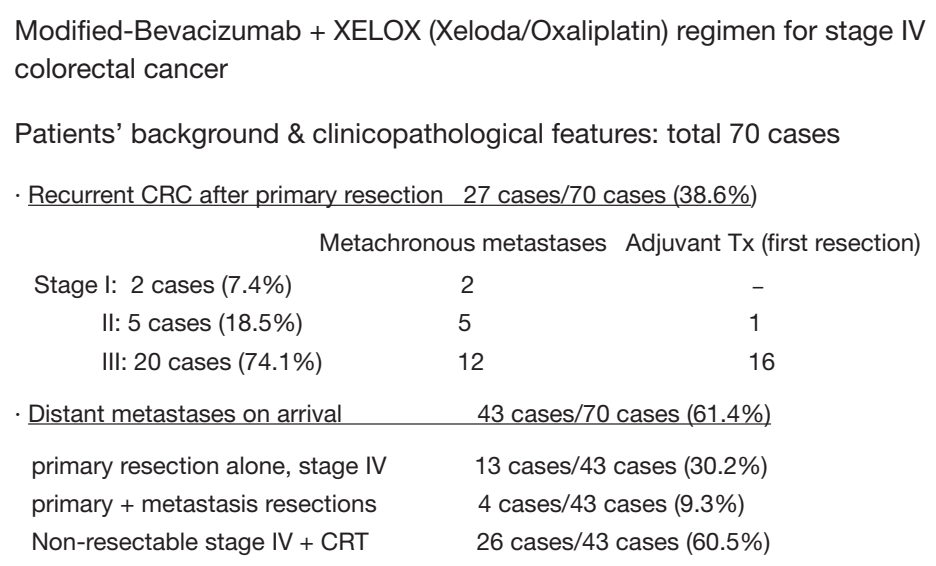

Figure 2 Background data and clinicopathological features of the 70 patients. 

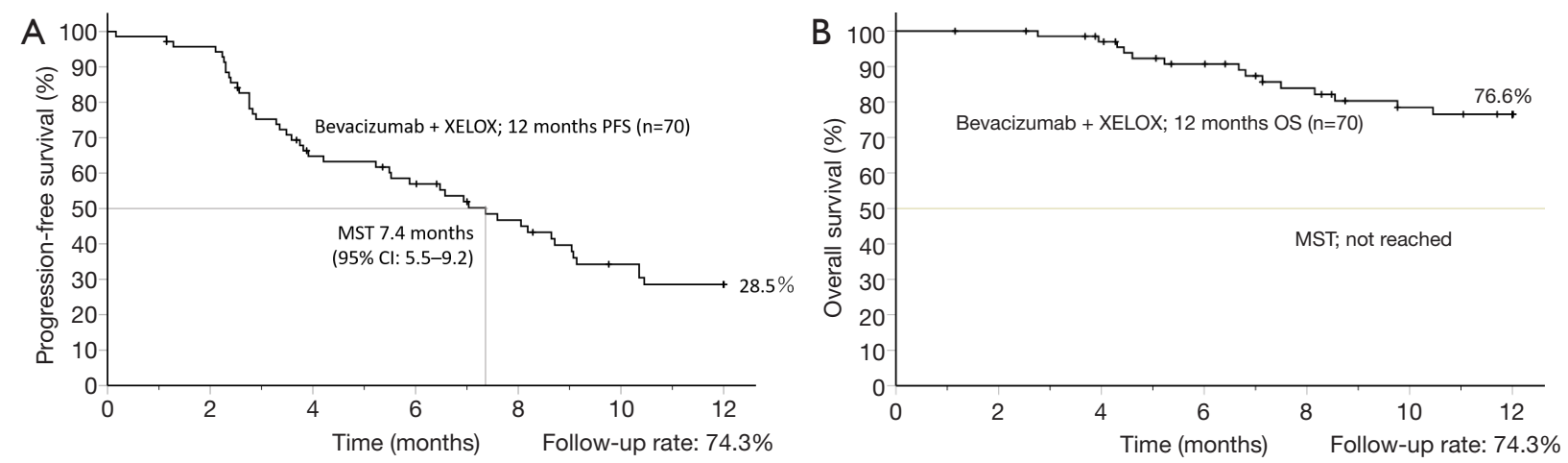

Figure 3 Kaplan-Meier curves. (A) 1Y-PFS was 28.5\% (MST: 7.4 months; 95\% CI: 5.5-9.2; follow-up rate: 74.3\%). (B) 1Y-OS was 76.6\% (MST not reached; follow-up rate: $74.3 \%$ ).
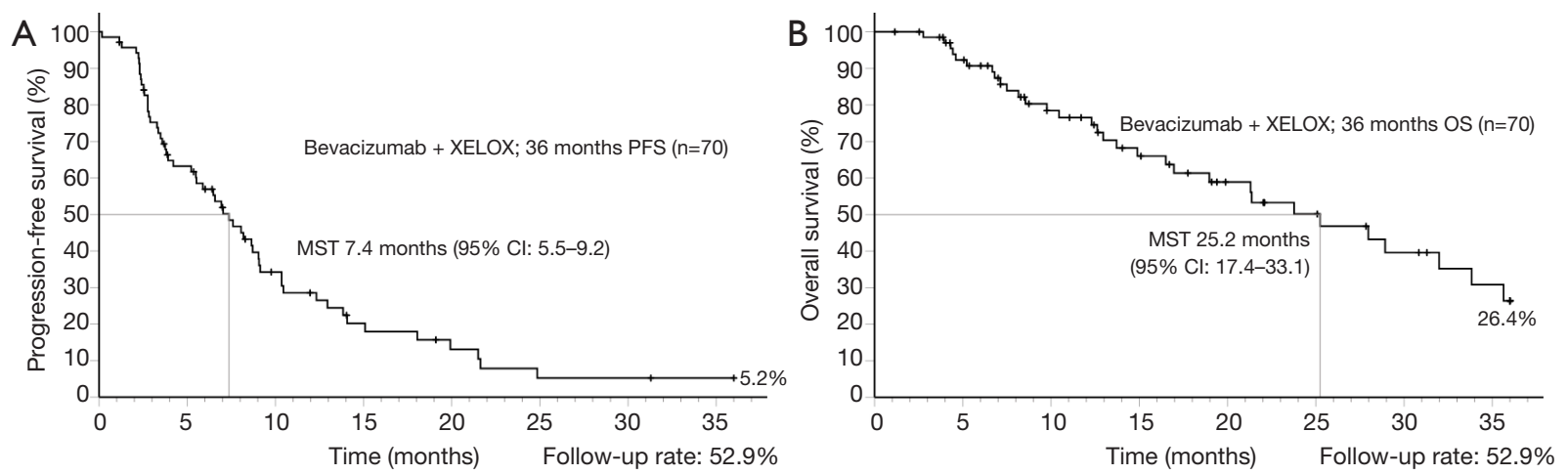

Figure 4 Kaplan-Meier curves. (A) 3Y-PFS was 5.2\% (MST: 7.4 months; 95\% CI: 5.5-9.2; follow-up rate: 52.9\%). (B) 3Y-OS was 26.4\% (MST: 25.2 months; 95\% CI: 17.4-33.1; follow-up rate: $52.9 \%$ ).

underwent R0/R1 resection of both the primary tumor and a metastatic lesion. Of these 4 patients, 2 patients had peritoneal dissemination, and 1 patient had multiple liver metastases. Twenty-six of these 43 patients $(60.5 \%)$ received chemoradiotherapy (CRT) (Figure 2).

\section{Outcomes}

(I) The survival rate was as follows: the 1Y-PFS was 28.5\% (MST: 7.4 months) (Figure 3A), and 1Y-OS was 76.6\% (MST: not reached) (Figure 3B). The 3Y-PFS was $5.2 \%$ (MST: 7.4 months) (Figure $4 A$ ) and $3 \mathrm{Y}-$ OS was 26.4\% (MST: 25.2 months) (Figure 4B). The mean and median number of treatment cycles was 13.1 and 10.5 , respectively.

(II) In terms of the objective tumor response, CR was not achieved, while PR was obtained in 20 patients (28.6\%), SD in 24 patients (34.3\%), and PD occurred in 26 patients $(37.1 \%)$. The objective response rate was $28.6 \%$ (20 patients), and the clinical benefit rate was $58.6 \%$ (41 patients) (Table 1). Dose reduction was required in 11 patients $(15.7 \%)$, including 3 patients with PR (27.3\%), 5 patients with SD (45.5\%), and 3 patients with PD $(27.3 \%)$. The dosing interval was prolonged in 41 patients $(58.6 \%)$, comprising 15 patients with PR (36.6\%)/ 16 patients with SD $(39.0 \%)$, and 10 patients with PD (24.4\%) (Table 1).

(III) The following adverse events were observed: palmar-plantar erythrodysesthesia syndrome (handfoot syndrome) in 52 patients $(74.3 \%$ ) (Grade 1 in 51 patients and Grade 2 in 1 patient), peripheral sensory neuropathy in 3 patients $(4.3 \%$ ) (Grade 1 in all 3 patients), leukopenia (white blood cell decreased) in 19 patients $(27.1 \%$ ) (Grade 1 in 15 patients, Grade 2 in 3 patients, and Grade 3 in 1 patient), hypertension in 66 patients $(94.3 \%$ ) (Grade 1 in 27 patients, 
Grade 2 in 35 patients, and Grade 3 in 4 patients; 21/66 patients had pre-existing hypertension that was being treated), diarrhea in 14 patients (20.0\%) (Grade 1 in 12 patients, Grade 2 in 1 patient, and Grade 3 in 1 patient), and nausea in 27 patients (38.6\%) (Grade 1 in 26 patients and Grade 2 in 1 patient]. All these adverse events resolved with conservative management (Table 2). A single Grade 4 event (perforation of the primary tumor) was observed in 1 patient $(1.4 \%)$ (Table 2).

(IV) Surgical conversion was achieved in 7 patients $(10 \%$ conversion rate). The resection sites in these patients were as follows: primary + liver in 2 patients, primary + lung in 1 patient, liver alone in 3 patients, and primary alone in 1 patient. The R0-CS rate was $71.4 \%$

Table 1 Objective tumor response

\begin{tabular}{|c|c|c|c|}
\hline Tumor response & All $(n=70)$ & $\begin{array}{l}\text { Dose } \\
\text { reduction rate } \\
(n=11,15.7 \%)\end{array}$ & $\begin{array}{l}\text { Dose-interval } \\
\text { prolongation rate } \\
(n=41,58.6 \%)\end{array}$ \\
\hline \multicolumn{4}{|c|}{ Best overall response, $\mathrm{n}(\%)$} \\
\hline $\mathrm{CR}$ & $0(0.0)$ & $0(0.0)$ & $0(0.0)$ \\
\hline PR & $20(28.6)$ & 3 (27.3) & $15(36.6)$ \\
\hline SD & 24 (34.3) & $5(45.5)$ & $16(39.0)$ \\
\hline PD & $26(37.1)$ & 3 (27.3) & $10(24.4)$ \\
\hline $\begin{array}{l}\text { Objective response } \\
\text { rate, } \mathrm{n}(\%)^{\star}\end{array}$ & 20 (28.6) & $3(27.3)$ & $15(36.6)$ \\
\hline $\begin{array}{l}\text { Clinical benefit rate, } \\
\mathrm{n}(\%)^{\#}\end{array}$ & $41(58.6)$ & $8(72.7)$ & 28 (68.3) \\
\hline
\end{tabular}

*, defined as $C R+P R ;$; , defined as $C R+P R+S D \geq 6$ months. $\mathrm{CR}$, complete response; $\mathrm{PR}$, partial response; $\mathrm{SD}$, stable disease; $\mathrm{PD}$, progressive disease.
(5/7 patients). The number of treatment cycles in patients receiving $\mathrm{R} 0$ conversion surgery ranged from 3 to 10 (mean: 7.3, median: 6.5). Their postoperative survival time ranged from 1 to 26 months (MST: 8 months).

\section{Discussion}

We previously reported the minimally effective cytotoxic dose (MECD) theory concerning the optimum dosages of chemotherapy agents for Japanese and elderly patients (21). Dosages of chemotherapy agents in western countries are usually based on the maximum-tolerated dose (MTD) and dose-limiting toxicity (DLT), which are determined from the results of multicenter randomized controlled trials $(22,23)$. The dose is calculated from the body surface area. However, Japanese people are smaller than Westerners. Japanese individuals with a height of $180 \mathrm{~cm}$ and a weight of $80 \mathrm{~kg}$ are not very common. Hence, regimens designed for western patients may not be tolerated, and suspension, dose reduction, or discontinuation of treatment is required in many patients (14-16). Accordingly, modified FOLFOX6 (oxaliplatin at $85 \mathrm{mg} / \mathrm{m}^{2}$ ) has become popular in Japan $(17,18)$ because it is associated with a lower incidence and grade of peripheral neuropathy, which is also more likely to resolve. In the present study, Grade 3-4 adverse events were not observed in palmar-plantar erythrodysesthesia syndrome (hand-foot syndrome) and peripheral sensory neuropathy. All the events observed were mild, and only one case of Grade 3 leukopenia was seen (1.4\%). For the IRIS regimen proposed in Japan, irinotecan is often administered at $80-100 \mathrm{mg} / \mathrm{m}^{2}$. Accordingly, oxaliplatin or irinotecan is administered at a dose of $85 \mathrm{mg} / \mathrm{m}^{2}$ in our Department. We use B-mab XELOX for the following reasons: (I) no

Table 2 Incidence and grade of adverse events according to CTCAE version 5 (JCOG)

\begin{tabular}{|c|c|c|c|c|}
\hline Adverse event & Grade 1, n (\%) & Grade 2, n (\%) & Grade 3, n (\%) & Grade 4, n (\%) \\
\hline Peripheral sensory neuropathy & $3(4.3)$ & $0(0.0)$ & $0(0.0)$ & $0(0.0)$ \\
\hline White blood cell decreased & $15(21.4)$ & $3(4.3)$ & $1(1.4)$ & $0(0.0)$ \\
\hline Hypertension & $27(38.6)$ & $35(50.0)$ & $4(5.7)$ & $0(0.0)$ \\
\hline Bleeding/perforation & $0(0.0)$ & $0(0.0)$ & $0(0.0)$ & $1(1.4)$ \\
\hline Diarrhea & $12(17.1)$ & $1(1.4)$ & $1(1.4)$ & $0(0.0)$ \\
\hline Nausea & $26(37.1)$ & $1(1.4)$ & $0(0.0)$ & $0(0.0)$ \\
\hline
\end{tabular}


need for reservoir implantation or extended continuous intravenous drip infusions, (II) short outpatient visits on days 1 and 15 of each cycle, and (III) oral treatment at home for 2 weeks (24). Dose reduction was required in only $15.7 \%$, whereas prolongation of the dosing interval was frequent and needed in $58.6 \%$. However, more than $80 \%$ of these patients could receive DIV every other week (days 1 and 15), followed by three weeks off without dose reduction (data not shown). For example, adjuvant XELOX for Stage III CRC patients at 6 months after surgery is oxaliplatin at $85 \mathrm{mg} / \mathrm{m}^{2}+85 \mathrm{mg} / \mathrm{m}^{2} / \times 6$ cycles over 24 weeks (total dose: $1,020 \mathrm{mg}$ ) at an outpatient clinic, rather than $130 \mathrm{mg} / \mathrm{m}^{2}$ of oxaliplatin every 3 weeks $\times 8$ cycles over 24 weeks (total dose: $1,040 \mathrm{mg}$ ). The modified/XELOX regimen is safe, and there is no dose suspension, dose reduction, or dropouts. Adverse events were mild. It seems that the modified dosages were not insufficient and were at least equivalent to the original regimen, taking into consideration the smaller body size of Japanese patients. Leukopenia was observed in 19 patients (27.1\%) (Grade 1 in 15 patients, Grade 2 in 3 patients, and Grade 3 in 1 patient), and the incidence of severe myelosuppression was low.

If severe myelosuppression occurs following chemotherapy, the patient may be admitted to receive granulocyte colony-stimulating factor (G-CSF). More than $90 \%$ of the patients in the present study had a P.S. of $0-2$, which allows outpatient management. Among the 19 patients with leukopenia, no patient required emergency admission or received G-CSF. A high QALY, high QOL, and long PFS and OS were considered to be achieved by B-mab XELOX therapy $(25,26)$.

Among patients with non-resectable advanced or recurrent Stage IV colorectal cancer without abdominal symptoms, systemic chemotherapy has recently been used as first-line therapy in patients with liver or lung metastasis. Subsequently, R0-CS is conducted if the tumor becomes macroscopically undetectable and R0 surgery is considered feasible $(11,12)$. A previous study showed that hepatic arterial infusion resulted in high response rates, and another reported that resection may be achieved in liver metastases that were previously unresectable $(27,28)$. However, recent advances in systemic chemotherapy indicate that the high response rates to hepatic arterial infusion do not affect long-term prognosis (29-31). Some colorectal cancer patients develop metastasis to the liver and/or lungs soon after resection of the primary tumor. In such patients, the response to chemotherapy should be evaluated, and the optimal timing for surgical resection of the metastatic lesions should be determined. However, to determine the optimum timing of conversion surgery, long-term studies are required to accumulate more data to better assess the relapse rate and incidence of metastasis. The conversion rate was low in the present series and only achieved in seven patients (10\%). In five of these patients (71.4\%) no macroscopic tumor was observed after surgery. Some of the study patients have been followed up for 4 years, and many have residual liver metastasis. These patients may benefit from multidisciplinary treatment; however, follow-up of more patients and for a more extended period is needed to obtain further data. Many unanswered questions remain, including the optimum number of adjuvant chemotherapy cycles, the adequacy of oral chemotherapy alone, and the appropriateness of discontinuing chemotherapy.

\section{Conclusions}

Bevacizumab in combination with XELOX showed promising OS in our study. Given the efficacy and toxicity data obtained in this study, it is possible that this treatment may become acceptable for Stage IV colorectal cancer patients in Japan.

\section{Acknowledgments}

This study was supported by Study Group (Tokai University Hachioji Hospital, Hachioji, Tokyo, Japan) and the Research and Study Program of Tokai University Educational System General Research Organization (approval no. 18R-310; Tokai University Hospital, Isehara, Kanagawa, Japan).

Funding: None.

\section{Footnote}

Reporting Checklist: The authors have completed the STROBE reporting checklist. Available at http://dx.doi. org/10.21037/jgo-20-350

Data Sharing Statement: Available at http://dx.doi. org/10.21037/jgo-20-350

Peer Review File: Available at http://dx.doi.org/10.21037/ jgo-20-350

Conflicts of Interest: All authors have completed the ICMJE uniform disclosure form (available at http://dx.doi. org/10.21037/jgo-20-350). The authors have no conflicts of 
interest to declare.

Ethical Statement: The authors are accountable for all aspects of the work in ensuring that questions related to the accuracy or integrity of any part of the work are appropriately investigated and resolved. The study was conducted in accordance with the Declaration of Helsinki (as revised in 2013). The study was approved by the Institutional Review Board (IRB) of Tokai University Medical School (IRB No. 18R-310) and individual consent for this retrospective analysis was waived.

Open Access Statement: This is an Open Access article distributed in accordance with the Creative Commons Attribution-NonCommercial-NoDerivs 4.0 International License (CC BY-NC-ND 4.0), which permits the noncommercial replication and distribution of the article with the strict proviso that no changes or edits are made and the original work is properly cited (including links to both the formal publication through the relevant DOI and the license). See: https://creativecommons.org/licenses/by-nc-nd/4.0/.

\section{References}

1. Fakih $M$ and Wong R. Efficacy of the monoclonal antibody EGFR inhibitors for the treatment of metastatic colorectal cancer. Curr Oncol 2010;17:S3-17.

2. Shankaran V, Obel J, Benson AB. Predicting response to EGFR inhibitors in metastatic colorectal cancer: current practice and future directions. Oncologist 2010;15:157-67.

3. Martinelli E, Troiani T, Morgillo F, et al. Emerging VEGF-receptor inhibitors for colorectal cancer. Expert Opin Emerg Drugs 2013;18:25-37.

4. Verdaguer H, Tabernero J, Macarulla T, et al. Ramucirumab in metastatic colorectal cancer: evidence to date and place in therapy. Ther Adv Med Oncol 2016;8:230-42.

5. Kong DH, Kim MR, Jang JH, et al. A review of antiangiogenic targets for monoclonal antibody cancer therapy. Int J Mol Sci 2017;18:E1786.

6. Lee GH, Malietzis G, Askari A, et al. Is right-sided colon cancer different to left-sided colorectal cancer? - a systematic review. Eur J Surg Oncol 2015;41:300-8.

7. Stintzing S, Tejpar S, Gibbs P, et al. Understanding the role of primary tumour localisation in colorectal cancer treatment and outcomes. Eur J Cancer 2017;84:69-80.

8. Lee MS, Menter DG, Kopetz S. Right versus left colon cancer biology: Integrating the consensus molecular subtypes. J Natl Compr Canc Netw 2017;15:411-9.

9. Arnold D, Lueza B, Douillard JY, et al. Prognostic and predictive value of primary tumour side in patients with RAS wild-type metastatic colorectal cancer treated with chemotherapy and EGFR directed antibodies in six randomized trials. Ann Oncol 2017;28:1713-29.

10. Boeckx N, Koukakis R, de Beeck KO, et al. Primary tumor sidedness has an impact on prognosis and treatment outcome in metastatic colorectal cancer: results from two randomized first-line panitumumab studies. Ann Oncol 2017;28;1862-8.

11. Cercek A, Goodman KA, Hajj C, et al. Neoadjuvant chemotherapy first, followed by chemoradiation and then surgery, in the management of locally advanced rectal cancer. J Natl Compr Canc Netw 2014;12:513-9.

12. Nozawa H, Ishihara S, Kawai K, et al. Conversion to resection in patients receiving systemic chemotherapy for unresectable and/or metastatic colorectal cancerPredictive factors and prognosis. Clin Colorectal Cancer 2018;17:e91-7.

13. Sato A, Doi T, Boku N, et al. Phase I/II Study of XELOX plus bevacizumab in Japanese patients with metastatic colorectal cancer (JO19380). Gan To Kagaku Ryoho 2011;38:561-9.

14. Saltz LB, Clarke S, Díaz-Rubio E, et al. Bevacizumab in combination with oxaliplatin-based chemotherapy as firstline therapy in metastatic colorectal cancer: A randomized Phase III study. J Clin Oncol 2008;26:2013-9.

15. Simkens LHJ, Tinteren HV, May A, et al. Maintenance treatment with capecitabine and bevacizumab in metastatic colorectal cancer (CAIRO3): a phase 3 randomised controlled trial of the Dutch Colorectal Cancer Group. Lancet 2015;385:1843-52.

16. Yamada $Y$, Denda T, Gamoh M, et al. S-1 and irinotecan plus bevacizumab versus mFOLFOX6 or CapeOX plus bevacizumab as first-line treatment in patients with metastatic colorectal cancer (TRICOLORE): a randomized, open-label, phase III, noninferiority trial. Ann Oncol 2018;29:624-31.

17. de Gramont A, Figer A, Seymour M, et al. Leucovorin and fluorouracil with or without oxaliplatin as first-line treatment in advanced colorectal cancer. J Clin Oncol 2000;18:2938-47.

18. Jeon HJ, Woo JH, Lee HY, et al. Adjuvant chemotherapy using the FOLFOX regimen in colon cancer. J Korean Soc Coloproctol 2011;27:140-6.

19. Eisenhauer EA, Therasse P, Bogaerts J, et al. New response evaluation criteria in solid tumours: revised RECIST 
guideline (version1.1). Eur J Cancer 2009;45:228-47.

20. DCTD, NCI, NIH, DHHS: Cancer Therapy Evaluation Program. Common terminology criteria for adverse events (CTCAE) v5.0. Available online: https://ctep.cancer. gov/protocolDevelopment/electronic_applications/docs/ CTCAE_v5_Quick_Reference_5x7.pdf

21. Mukai M, Tajima T, Hasegawa S, et al. Recent molecular immunological chemotherapy for gastrointestinal malignancies. Clin Oncol 2017;2:E1233.

22. Khayat D, Gil-delgado M, Antoine EC, et al. The role of irinotecan and oxaliplatin in the treatment of advanced colorectal cancer. Oncology 2001;15:415-29; discussion 429-30, 433-4.

23. Saif $M W$ and Reardon J. Management of oxaliplatininduced peripheral neuropathy. Ther Clin Risk Manag 2005;1:249-58.

24. Muro K, Boku N, Shimada Y, et al. Irinotecan plus S-1 (IRIS) versus fluorouracil and folinic acid plus irinotecan (FOLFIRI) as second-line chemotherapy for metastatic colorectal cancer: a randomized phase $2 / 3$ non-inferiority study (FIRIS study). Lancet Oncol 2010;11:853-60.

25. Kirkdale R, Krell J, Brown CO, et al. The cost of a QALY. QJM 2010;103:715-20.

26. Antonuzzo L, Giommoni E, Pastorelli D, et al.

Cite this article as: Yokoyama D, Mukai M, Uda S, Kishima K, Koike T, Hasegawa S, Izumi H, Yamamoto S, Tajima T, Nomura E, Makuuchi H. Efficacy of modified bevacizumabXELOX therapy in Japanese patients with stage IV recurrent or non-resectable colorectal cancer. J Gastrointest Oncol 2021;12(2):527-534. doi: 10.21037/jgo-20-350
Bevacizumab plus XELOX as first-line treatment of metastatic colorectal cancer: The OBELIX study. World J Gastroenterol 2015;21:7281-8.

27. Arai Y, Inaba Y, Takeuchi Y, et al. Intermittent hepatic arterial infusion of high-dose 5-FU on a weekly schedule for liver metastases from colorectal cancer. Cancer Chemother Pharmacol 1997;40:526-30.

28. Bismuth H, Adam R, Lévi F, et al. Resection of nonresectable liver metastases from colorectal cancer after neoadjuvant chemotherapy. Ann Surg 1996;224:509-20.

29. Hohn DC, Stagg RJ, Friedman MA, et al. A randomized trial of continuous intravenous versus hepatic intraarterial floxuridine in patients with colorectal cancer metastatic to the liver: The Northern California Oncology Group trial. J Clin Oncol 1989;7:1646-54.

30. Martin JK, O’Connell MJ, Wieand HS, et al. Intra-arterial floxuridine vs systemic fluorouracil for hepatic metastases from colorectal cancer. A randomized trial. Arch Surg 1990;125:1022-7.

31. Kemeny N, Daly J, Reichman B, et al. Intrahepatic or systemic infusion of fluorodeoxyuridine in patients with liver metastases from colorectal carcinoma. A randomized trial. Ann Int Med 1987;107:459-65. 